Service social

\title{
Mutual Aid - A Factor of Evolution, par Peter Koropotkin, Montréal, Black Rose Books, 1989, 363 pages.
}

\section{Jocelyn Lindsay}

Volume 39, numéro 1, 1990

Le groupe ici et ailleurs

URI : https://id.erudit.org/iderudit/706471ar

DOI : https://doi.org/10.7202/706471ar

Aller au sommaire du numéro

Éditeur(s)

École de service social de l'Université Laval

ISSN

1708-1734 (numérique)

Découvrir la revue

Citer ce compte rendu

Lindsay, J. (1990). Compte rendu de [Mutual Aid - A Factor of Evolution, par Peter Koropotkin, Montréal, Black Rose Books, 1989, 363 pages.] Service social, 39(1), 193-196. https://doi.org/10.7202/706471ar d'utilisation que vous pouvez consulter en ligne.

https://apropos.erudit.org/fr/usagers/politique-dutilisation/ 
De plus, une thérapie s'étendant sur dix-huit mois, me paraît un luxe que nos organismes surchargés ne pourraient offrir à seulement quatre enfants. Le soin apporté par les auteurs à décrire le programme proposé dans ses moindres détails, aidera sûrement toute nouvelle équipe à entreprendre le traitement sans perte de temps et à parvenir systématiquement au but proposé. J'opterais facilement pour une année scolaire pleinement utilisée, quitte à ce qu'un ou deux des membres du groupe soient réadmis dans le groupe de l'année suivante, si besoin s'en faisait sentir.

La seconde partie du document détaille chacun des objectifs d'intervention en sous-objectifs et propose des activités pour leur mise en œuvre. Ce programme a fait l'objet d'une très minutieuse préparation facilitant ainsi son utilisation par tout nouvel intervenant, qui pourrait tout aussi bien remplacer les activités proposées sans déroger au but ultime, puisque les objectifs sont clairement identifiés.

Une thérapie pour ce genre d'enfants peut prendre des proportions énormes, considérant qu'il y a tant à faire. Les auteurs ont évité ce problème en considérant deux types d'objectifs. Les uns, séquentiels, s'attaquent aux problèmes engendrés par l'abus et permettent d'arriver, par étapes successives, au but projeté. Les autres, linéaires, visent à orienter l'enfant vers une nouvelle phase de maturité, en répondant à ses besoins personnels et ces objectifs peuvent se poursuivre au-delà de la thérapie de groupe.

En résumé, ce document propose un modèle d'intervention très structuré, en dépit de l'appellation d'approche semi-structurée que lui donnent ses auteurs. Comme ce programme a été préparé avec beaucoup de soin, mis à l'essai et corrigé, il sera d'un grand secours à tout organisme qui voudra implanter un nouveau type d'intervention pour une clientèle d'enfants victimes d'abus sexuels. Nous recommanderions simplement à un intervenant chevronné de faire preuve de plus de latitude dans le choix des activités thérapeutiques afin d'éviter une répétition qui lasserait les jeunes.

Nous supposons également qu'un travail parallèle est entrepris avec les parents, puisque l'objectif ultime est le renouement de la famille; aussi les rencontres avec l'abuseur et avec la famille auraient sûrement gagné à être mieux développées dans le présent guide.

\section{Céline Bédard}

École de service social Université Laval

\section{Mutual Aid - A Factor of Evolution, par Peter KROPOTKIN, Montréal, Black Rose Books, 1989, 362 pages.}

Il y a des livres qui passent rapidement au firmament de la popularité, d'autres pour lesquels le temps ne fait que confirmer leur pleine valeur. Mutual Aid appartient nettement à la deuxième catégorie. Cet ouvrage, qui est le principal de Kropotkin, est connu en de nombreuses disciplines; traduit en plusieurs langues, il a toujours été accessible en anglais, depuis plus de huit décennies*.

De fait, il est assez exceptionnel que des travailleurs sociaux de groupe puissent s'intéresser aux écrits d'un géographe russe du siècle dernier. C'est dans de nombreux écrits de William Schwartz et de Laurence Shulman que j'ai trouvé les premières références à l'œuvre de Kropotkin, plus précisément par rapport aux fondements de l'aide mutuelle. Mais il faut d'abord replacer cet auteur dans son contexte. 
Né en 1842 et issu d'une famille noble de la Russie, Kropotkin a choisi, après des études militaires et scientifiques, d'aller œuvrer comme cosaque en Sibérie, là où il pourrait mener à bien ses recherches. Dans de nombreux voyages d'études, d'abord comme cosaque puis comme secrétaire d'une section de la Société russe de géographie, il s'est livré à de multiples observations attentives des espèces animales.

II faut aussi savoir que ses observations et ses écrits se sont voulus un contre-argument à la philosophie dominante de l'époque (représentée particulièrement par Alfred Russel Wallace et par Thomas Huxley dont on retrouve la position en appendice B), selon laquelle la lutte pour l'existence est le facteur premier de l'évolution. Kropotkin a donc voulu montrer qu'au-delà de la loi de la lutte, il y a la loi de l'aide mutuelle, beaucoup plus importante au plan de l'évolution progressive de l'espèce.

À côté de l'observateur scientifique, Kropotkin était aussi un théoricien et un militant de l'anarchisme (croyance en la coopération naturelle des individus et refus systématique de tout pouvoir établi dont celui de l'État, qui détruit cette tendance en jugulant les initiatives personnelles sous le couvert de la normalisation).

Cela devait l'amener à être emprisonné en 1874, puis à s'exiler deux ans plus tard en Angleterre. De fait, c'est surtout dans ce pays et en France qu'il vécut le reste de sa vie, avant de retourner finir ses jours en Russie où il mourut en 1921. Pendant son long passage en Occident, marqué de quelques emprisonnements sous l'influence du gouvernement russe, il a produit la majorité de ses œuvres en ayant le support des communautés scientifiques française et anglaise.

Ce livre a d'abord été produit en pièces détachées : il s'agit d'une série d'articles s'étendant sur sept ans (1890-1896) qui ont ensuite été réunis et dont la première édition complète date de 1902. L'ouvrage a donc été écrit pour être accessible à tous, et l'auteur y a développé les implications de la théorie de l'aide mutuelle aux niveaux animal et humain, en montrant que ses propositions sont fondées sur des constantes à l'intérieur des différentes espèces.

La présente édition du volume commence par une présentation écrite par George Woodstock, qui situe la vie et l'œuvre de Kropotkin.

On retrouve dans l'introduction une définition de l'aide mutuelle : " $C^{\prime}$ est un sentiment infiniment plus large que l'amour ou la sympathie personnelle, un instinct qui s'est peu à peu développé parmi les animaux et les hommes au cours d'une évolution extrêmement lente, et qui a appris aux animaux comme aux hommes la force qu'ils pouvaient trouver dans la pratique de l'entraide et du soutien mutuel, ainsi que les plaisirs que pouvait leur donner la vie sociale» (p. XLi).

L'auteur procède d'abord à un examen de la vie collective à l'intérieur du règne animal, notamment chez les fourmis, les abeilles, les oiseaux et divers mammifères. S'inspirant des travaux de plusieurs autres naturalistes, il montre que la sociabilité à l'intérieur d'une espèce animale est un phénomène très répandu à tous les niveaux, de telle sorte qu'on peut considérer cela comme une loi de la nature. Autant les espèces qui ne font pas preuve d'un comportement sociable sont condamnées à dépérir, autant celles qui ont développé I'habitude de l'entraide et de la protection mutuelle vont assurer la conservation de l'espèce et son évolution.

La même argumentation s'applique à l'homme primitif, qui doit sa position spéciale par rapport aux espèces animales aux aptitudes et aux 
habiletés cultivées en société. L'intelligence est ici vue comme une faculté éminemment sociale, nourrie par le langage et par les autres formes de communication, par l'imitation et par le cumul de l'expérience. L'homme primitif ne vit pas dans des familles mais dans des tribus où la loi formelle n'existe pas, et où un ensemble de coutumes assurent une aide mutuelle.

Dans les clans et dans les tribus, I'homme avait déjà développé un grand nombre d'institutions sociales. C'est dans les tribus sauvages que la commune villageoise s'est développée, avec comme point de départ la possession en commun d'un territoire et sa défense, avec comme autorité l'assemblée à l'intérieur de chacun des villages, lesquels sont regroupés en fédérations.

Kropotkin montre aussi la richesse de la vie communautaire dans les cités du Moyen-Âge. Compte tenu du développement des connaissances, ces cités se sont appuyées sur l'unité territoriale et sur les guildes ou fraternités, constituées de personnes formées pour exercer un art ou un métier spécifiques.

S'attardant aux derniers siècles du développement de l'humanité, l'auteur montre que le développement de l'État sur le modèle de la Rome impériale a brutalement stoppé l'évolution des institutions d'entraide au Moyen-Âge. Mais la tendance à l'entraide est d'une telle force qu'elle s'affirme "de nouveau dans une infinité d'associations qui tendent maintenant à englober toutes les manifestations de la vie sociale et à prendre possession de tout ce dont l'homme a besoin pour vivre et pour réparer les pertes causées par la vie " (p. 294). Et malgré l'acharnement de l'État à détruire la vie commune, il observe l'émergence de syndicats, de coopératives, d'associations, de clubs et de sociétés. Et de conclure Kropotkin : " Le besoin d'entraide et d'appui mutuel qui avait trouvé un dernier refuge dans le cercle étroit de la famille, ou parmi les voisins des quartiers pauvres des grandes villes, dans les villages ou dans les associations secrètes d'ouvriers, s'affirme à nouveau dans notre société moderne elle-même et revendique son droit d'être, comme il l'a toujours été, le principal facteur du progrès» (p. 292).

L'analyse d'un tel volume déborde les cadres des critiques habituelles, puisqu'il transcende plusieurs disciplines comme la géographie, l'histoire, la biologie, I'anthropologie et la sociologie.

Ce livre est écrit dans un langage simple, qui évite la terminologie scientifique obscure. II est encore considéré comme un fondement important de la théorie anarchiste et de l'étude de l'écologie sociale. Il invite aussi à une nouvelle lecture de I'histoire qui, " telle qu'elle a été écrite jusqu'à présent, n'est pour ainsi dire qu'une description des voies et moyens par lesquels la théocratie, le pouvoir militaire, l'autocratie et plus tard la plutocratie ont été amenés, établis et maintenus» (p. 295).

En relation au travail social de groupe, j'ai l'impression que la lecture de cet ouvrage permet une compréhension beaucoup plus globale de l'aide mutuelle, qui n'apparaît plus comme un phénomène restreint, mais comme une force qui outrepasse l'espèce humaine, qui refait surface chaque fois qu'on l'oublie et qui se développe dans des formes de plus en plus avancées. On est ici très loin d'un état passager à favoriser par quelques artifices, et je comprends mieux l'insistance de Shulman lorsqu'il suggère d'enlever les obstacles à l'aide mutuelle, laquelle est une force, une tendance, un principe si fondamental dans la pratique auprès des groupes. 


\section{Note}

* Nous avons eu accès à une version française, datant de 1938 (L'entr'aide, Paris, Alfred Costes éditeur).

Proceedings - 1989 - Actes du symposium (Volumes 1 et 2). Publiés par le Comité de coordination du $11^{\mathrm{e}}$ symposium annuel de I'Association for the Advancement of Social Work with Groups, avec la collaboration de Jocelyn Lindsay et Jean-Pierre Landriault, 1392 pages.

Les Actes regroupent les textes de 81 communications présentées au symposium sur le service social des groupes tenu à Montréal du 26 au 29 octobre 1989.

Les articles - quelques-uns en français mais la majorité en anglais - sont regroupés par sections. Une première division intitulée "Plénières" contient la conférence de Margot Breton ainsi que celle de Beulah Rothman et Catherine Papell. Étant donné l'importance de leur contenu respectif pour l'enseignement et la pratique actuels de l'intervention de groupe, ces conférences ont été traduites pour Service social et reproduites dans le présent numéro sous les titres suivants : "Leçons à tirer de nos traditions en service social des groupes" (p. 13-26); "Service social des groupes en contexte d'autorité " (p. 45-63). Une deuxième section, intitulée " Administration » (p. 47-60) traite de trois aspects à prendre en considération dans la préparation et la réalisation d'un programme de groupe : les difficultés reliées à l'implantation et au développement des groupes, certaines conditions d'efficacité (dont le nombre de membres et d'intervenants, la durée du groupe et les divers frais à prévoir) en rapport avec les coûts du programme, la formation des intervenants et la coanimation. Même si les auteurs, S.S. Korman et D.S. Donofrio, appuient leur réflexion sur leur propre expérience dans un organisme qui offre des groupes de thérapie aux enfants et adolescents émotionnellement perturbés ainsi qu'à leur famille, leurs commentaires demeurent applicables à tout programme de groupe.

Les Actes présentent ensuite huit sections thématiques: personnes âgées; groupes populaires et mouvements sociaux; famille, enfance et adolescence; femmes et hommes; groupes avec besoins spéciaux; santé, santé mentale, personnes handicapées; groupes minoritaires, milieu multiculturel et international; théorie, formation et recherche.

Les différents sujets présentés dans les Actes témoignent de la diversité et de l'étendue de la méthode de groupe. On y discute d'intervention auprès de clientèles très diversifiées, partant des jeunes enfants, des adolescents et de leurs parents, jusqu'aux adultes et aux personnes âgées. De nombreuses problématiques y sont aussi abordées : besoins de répit chez les donneurs de soins aux personnes âgées ou handicapées, problèmes des familles monoparentales, des personnes séparées ou divorcées, des familles en transition, des familles recomposées, des personnes handicapées, des sidéens, des psychiatrisés. On y traite aussi d'isolement social, de violence, de toxicomanie, de délinquance juvénile, d'abus sexuels, etc. L'intervention auprès 